\title{
THE CONVERGENCE OF RATIONAL FUNCTIONS OF BEST APPROXIMATION TO THE EXPONENTIAL FUNCTION
}

\author{
BY
}

E. B. SAFF

\begin{abstract}
The object of the paper is to establish convergence throughout the entire complex plane of sequences of rational functions of prescribed types which satisfy a certain degree of approximation to the function $a e^{\gamma z}$ on the disk $|z| \leqq \rho$. It is assumed that the approximating rational functions have a bounded number of free poles. Estimates are given for the degree of best approximation to the exponential function by rational functions of prescribed types. The results obtained in the paper imply that the successive rows of the Walsh array for $a e^{y z}$ on $|z| \leqq \rho$ converge uniformly to $a e^{y z}$ on each bounded subset of the plane.
\end{abstract}

1. Introduction. A rational function $r_{n v}(z)$ of the complex variable $z$ is said to be of type $(n, v)$ if it can be expressed in the form

$$
r_{n v}(z)=\frac{a_{0} z^{n}+a_{1} z^{n-1}+\cdots+a_{n}}{b_{0} z^{\nu}+b_{1} z^{\nu-1}+\cdots+b_{v}}, \quad \sum_{0}^{v}\left|b_{k}\right| \neq 0
$$

where the $a_{i}, b_{i}$ are complex constants. The possibility that $a_{0}=0$ or $b_{0}=0$ is not excluded so that a rational function which is of type $(n, \nu)$ is also of type $(m, \mu)$ if $m \geqq n$ and $\mu \geqq \nu$.

Let the function $f(z)$ be continuous on the closed disk $\Delta_{\rho}:|z| \leqq \rho$. Then for each pair of nonnegative integers $(n, v)$ there exists a rational function $W_{n v}(z)$ of type $(n, v)$ which is of best Tchebycheff (uniform) approximation to $f(z)$ on $\Delta_{\rho}$ in the sense that the inequality

$$
E_{n v}(f ; \rho) \equiv\left[\max \left|f(z)-W_{n v}(z)\right| ; z \text { on } \Delta_{\rho}\right] \leqq\left[\max \left|f(z)-r_{n v}(z)\right| ; z \text { on } \Delta_{\rho}\right]
$$

holds for every rational function $r_{n v}(z)$ of type $(n, v)$. Best approximating rational functions need not be unique $[1, \S 12.4]$ but any particular determination of them

Presented to the Society, January 23, 1970; received by the editors December 29, 1969 and, in revised form, February 17, 1970.

AMS 1969 subject classifications. Primary 4117, 3070.

Key words and phrases. Free poles, rational functions of type $(n, v)$, best approximating rational functions, Walsh array, Padé table, polynomial of least squares approximation, generalized Bernstein lemma. 
will suffice for our purposes. The $W_{n v}(z)$ form a table of double entry

$$
\begin{array}{cccc}
W_{00}(z) & W_{10}(z) & W_{20}(z) & \ldots \\
W_{01}(z) & W_{11}(z) & W_{21}(z) & \ldots \\
W_{02}(z) & W_{12}(z) & \ldots & \ldots
\end{array}
$$

known as the $L_{\infty}$ Walsh array [2] for $f(z)$ on $\Delta_{\rho}$. The first row of the array, which consists of best approximating polynomials, has been the object of considerable study. Comparatively little is known, however, of the behavior of the other rows of the array or of other sequences of rational functions which may be formed from the array.

If the function $f(z)$ is analytic on $\Delta_{\rho}$ and meromorphic with precisely $\nu$ poles in the open disk $|z|<\sigma, \rho<\sigma \leqq \infty$, J. L. Walsh has shown [3, p. 3] that the $(\nu+1)$ th row of his array converges to $f(z)$ at each point of $|z|<\sigma$. In a recent paper [4] the author established the convergence of certain other rows of the array for the case where $f(z)$ has a multiple pole on $|z|=\sigma$. However, the two results mentioned yield no information about the convergence of the successive rows of the Walsh array for a function $f(z)$ which is entire and transcendental.

It is the aim of the present paper to establish the first such convergence theorem, namely, for the function $f(z)=e^{z}$. In $\S 2$ we prove that for fixed $\nu$ and $n$ sufficiently large each of the rational functions $W_{n \nu}(z)$ of best approximation to $e^{z}$ on $\Delta_{\rho}$ has precisely $\nu$ finite poles and these poles approach infinity. Consequently we deduce that every row of the Walsh array for $e^{z}$ on $\Delta_{\rho}$ converges uniformly to $e^{z}$ on each bounded subset of the plane. Analogous convergence theorems are then established for the columns of the Walsh array. To prove these results it is first shown (compare [5, p. 168]) that for fixed $\nu$ the degree of best rational approximation $E_{n v}\left(e^{z} ; \rho\right)$ to $e^{z}$ on $\Delta_{\rho}$ satisfies

$$
0<A_{1} \leqq(n+2 \nu+1) ! \rho^{-n} E_{n v}\left(e^{z} ; \rho\right) \leqq A_{2}<\infty, \quad n \geqq 0 .
$$

In $\S 3$ we establish the convergence of sequences of rational functions which are of least $p$ th power line integral approximation to $e^{z}$ on $|z|=\rho$. The results obtained here and in $\$ 2$ are stated not merely for best approximating rational functions but for any sequence of rational functions of prescribed types which satisfy a certain degree of approximation to $e^{z}$.

The Walsh array is analogous to the much applied table of Padé $[6, \S 73]$, which consists of interpolating rational functions. The Padé rational function of type $(n, v)$ for the function $e^{z}$ is defined to be the rational function $R_{n v}(z)$ with the property that among all rational functions of type $(n, v)$ the difference $e^{z}-R_{n v}(z)$ has a zero of highest order at $z=0$. We shall write $R_{n v}(z)=P_{n v}(z) / Q_{n v}(z)$, where $P_{n v}(z)$ is a polynomial of degree $n, Q_{n v}(z)$ is a polynomial of degree $\nu$, and $Q_{n v}(0)=1$. Padé established [6, p. 434] a convergence theorem for the $R_{n v}(z)$ which, in partic- 
ular, implies that for fixed $\nu$ we have $\lim _{n \rightarrow \infty} Q_{n v}(z)=1$ and $\lim _{n \rightarrow \infty} R_{n v}(z)=e^{z}$ uniformly on each compact subset of the plane. This result plays an important part in the proofs to follow.

2. Rational functions of Tchebycheff approximation. To establish the inequalities (1) we prove two lemmas.

LEMMA 1. Let $R^{v}$ be the set of all ordered $\nu$-tuples $x=\left(x_{1}, x_{2}, \ldots, x_{v}\right)$ of real numbers and let

$$
L_{n, k}(x) \equiv 1 /(n+k) !+\sum_{i=1}^{\nu} x_{i} /(n+k-i) ! .
$$

If $1 \leqq \mu \leqq \nu+1$, then for each $n(\geqq \nu)$ the system of $\nu$ linear equations $L_{n, k}(x)=0$, $1 \leqq k \leqq \nu+1, k \neq \mu$, has a unique solution $x_{n \mu}$ and

$$
\left|L_{n, \mu}\left(x_{n \mu}\right)\right|=(\mu-1) !(\nu+1-\mu) ! n ! /(n+\mu-1) !(n+\nu+1) ! .
$$

Proof. Let $D_{n, \nu}^{(\mu)}$ be the determinant of the matrix $A_{n, \nu}^{(\mu)}$ obtained by deleting the $\mu$ th row from the $(\nu+1)$-by- $\nu$ matrix

$$
\left[\begin{array}{cccc}
1 / n ! & 1 /(n-1) ! & \cdots & 1 /(n+1-\nu) ! \\
1 /(n+1) ! & 1 / n ! & \cdots & 1 /(n+2-\nu) ! \\
\cdot & \cdot & & \cdot \\
\cdot & \cdot & & \cdot \\
\cdot & \cdot & & \cdot \\
1 /(n+\nu) ! & 1 /(n+\nu-1) ! & \cdots & 1 /(n+1) !
\end{array}\right]
$$

It is known $[9, \S 769]$ that

$$
D_{n, v}^{(1)}=\prod_{i=1}^{v} \frac{(\nu-i) !}{(n+i) !}
$$

and the same method used to derive this formula can be used to show that for $1<\mu<\nu+1$ we have

$$
D_{n, \nu}^{(\mu)}=\frac{D_{n+\mu-1, v-\mu+1}^{(1)}}{(\mu-1) !} \prod_{i=0}^{\mu-2} \frac{(\nu-i) !}{(n+i) !}
$$

Indeed, if from the $j$ th column $(j=2, \ldots, \nu)$ of $A_{n, v}^{(\mu)}, 1<\mu$, we subtract the $(j-1)$ th column multiplied by the factor $n-j+2$, then we obtain

$$
D_{n, v}^{(\mu)}=\nu ! D_{n+1, \nu-1}^{(\mu-1)} / n !(\mu-1) .
$$

Repeated application of the reduction formulae (5) then leads to (4). Since $D_{n, v}^{(\nu+1)}=D_{n-1, v}^{(1)}$ it is clear that no $D_{n, v}^{(\mu)}$ is zero and hence the system $L_{n, k}(x)=0$, $1 \leqq k \leqq \nu+1, k \neq \mu$, has a unique solution $x_{n \mu}$. 
It is easy to verify that

$$
L_{n, \mu}\left(x_{n \mu}\right)=\frac{1}{D_{n, \nu}^{(\mu)}}\left|\begin{array}{cccc}
1 /(n+\mu) ! & 1 /(n+\mu-1) ! & \ldots & 1 /(n+\mu-\nu) ! \\
1 /(n+1) ! & 1 / n ! & \cdots & 1 /(n+1-\nu) ! \\
\cdot & \cdot & & \cdot \\
\cdot & \cdot & & \cdot \\
1 /(n+\mu-1) ! & 1 /(n+\mu-2) ! & \cdots & 1 /(n+\mu-1-\nu) ! \\
1 /(n+\mu+1) ! & 1 /(n+\mu) ! & \cdots & 1 /(n+\mu+1-\nu) ! \\
\cdot & \cdot & & \cdot \\
\cdot & \cdot & & \cdot \\
\cdot & \cdot & & \cdot \\
1 /(n+\nu+1) ! & 1 /(n+\nu) ! & \cdots & 1 /(n+1) !
\end{array}\right|,
$$

and hence $\left|L_{n, \mu}\left(x_{n \mu}\right)\right|=D_{n, v+1}^{(1)} / D_{n, v}^{(\mu)}$. Using equations (3) and (4) the last expression may be evaluated to obtain (2) which completes the proof.

LEMMA 2. The function $f_{n}(x) \equiv \sum_{k=1}^{v+1}\left|L_{n, k}(x)\right|, x \in R^{\nu}, n \geqq v$, has an absolute minimum at the point $x_{n, v+1}$ so that

$$
f_{n}(x) \geqq \nu ! n ! /(n+\nu) !(n+\nu+1) !, \quad x \in R^{\nu} .
$$

Proof. We first show that $f_{n}(x)$ has an absolute minimum in $R^{v}$. Let $m \equiv$ $\inf \left\{f_{n}(\boldsymbol{x}) \mid \boldsymbol{x} \in R^{\nu}\right\}$ and let $S \equiv \bigcap_{k=1}^{v+1}\left\{\boldsymbol{x}|| L_{n, k}(\boldsymbol{x}) \mid \leqq m+1\right\}$. Since $f_{n}(\boldsymbol{x})>m+1$ for $x \notin S$ we have that $m=\inf \left\{f_{n}(x) \mid x \in S\right\}$. Note that the set $S$ is a closed convex polyhedron. Furthermore $S$ must be bounded, for the contrary assumption implies that $S$ contains a ray, i.e., there exists a vector $u \in S$ and a nonzero vector $v$ such that $u+\lambda v \in S$ for every $\lambda \geqq 0$. Since the homogeneous system $L_{n, k}(x)-1 /(n+k) !=0$, $1 \leqq k \leqq \nu+1$, has only the trivial solution, there exists an integer $k^{*}, 1 \leqq k^{*} \leqq \nu+1$, for which $b \equiv L_{n, k^{*}}(v)-1 /\left(n+k^{*}\right) ! \neq 0$. But then we have

$$
\left|L_{n, k^{*}}(u+\lambda v)\right|=\left|L_{n, k^{*}}(u)+\lambda b\right| \leqq m+1,
$$

for all $\lambda \geqq 0$ which is impossible. Thus $S$ is bounded and since $S$ is also closed the continuous function $f_{n}(\boldsymbol{x})$ attains its absolute minimum $m$ on $S$.

Now let $\mathscr{M} \equiv\left\{\boldsymbol{x} \in R^{v} \mid f_{n}(\boldsymbol{x})=m\right\}$ and we show that there exists a vector in $\mathscr{M}$ which satisfies $\nu$ of the equations $L_{n, k}(x)=0, k \in I \equiv\{1,2, \ldots, \nu+1\}$. For each $x \in \mathscr{M}$ put $I(x) \equiv\left\{k \in I \mid L_{n, k}(x)=0\right\}$ and let $x^{*} \in \mathscr{M}$ be a vector for which $I\left(x^{*}\right)$ has maximum order. It is clear from Lemma 1 that the set $J \equiv I-I\left(x^{*}\right)$ is nonempty so that $x^{*}$ is an interior point of the closed polyhedron

$$
K_{1} \equiv \bigcap_{k \in J}\left\{x \in R^{v} \mid L_{n, k}\left(x^{*}\right) L_{n, k}(x) \geqq 0\right\}
$$


If $I\left(x^{*}\right)$ is nonempty, we set

$$
K_{2} \equiv \bigcap_{k \in I\left(x^{*}\right)}\left\{x \in R^{v} \mid L_{n, k}(x)=0\right\}
$$

Otherwise let $K_{2} \equiv R^{v}$. Now note that on the set $K \equiv K_{1} \cap K_{2}$ the function $f_{n}(x)$ is the sum of a linear function plus a constant and hence is concave in $K$. Since $x^{*}$ is an interior point of $K_{1}$, it is easy to see that for each vector $y \in K$ there is a vector $w \in K$ and a scalar $\delta, 0<\delta<1$, such that $x^{*}=\delta y+(1-\delta) w$. Thus from the concavity of $f_{n}(\boldsymbol{x})$ we deduce as in [7, p. 194] that $f_{n}(\boldsymbol{x})=m$ for every $\boldsymbol{x} \in K$. Since the order of $I\left(x^{*}\right)$ is maximal, we therefore have $L_{n, k}\left(x^{*}\right) L_{n, k}(x)>0$ for $x \in K$ and $k \in J$. Hence $K=K_{1}^{0} \cap K_{2}$, where

$$
K_{1}^{0} \equiv \bigcap_{k \in J}\left\{x \in R^{v} \mid L_{n, k}\left(x^{*}\right) L_{n, k}(x)>0\right\},
$$

and so the set $K$ is both open and closed relative to $K_{2}$. Consequently $K=K_{2}$ and we deduce that $K_{2} \subset K_{1}^{0}$. Now if the order of $I\left(x^{*}\right)$ is strictly less than $\nu$, then at least one of the points $\boldsymbol{x}_{n \mu}$ of Lemma 1 lies in $K_{2}$ but not in $K_{1}^{0}$. Thus $\boldsymbol{x}^{*}$ must be equal to one of the points $x_{n \mu}$ and from (2) it is easy to see that $\boldsymbol{x}^{*}=\boldsymbol{x}_{n, v+1}$ which completes the proof.

We may now prove

THEOREM 1. Let $a$ and $\gamma$ be nonzero complex numbers. For fixed $v(\geqq 0)$ the degree of best rational approximation $E_{n v}\left(a e^{\gamma z} ; \rho\right)$ to the function ae $e^{\gamma z}$ on the disk $\Delta_{\rho}$ satisfies

$$
0<A_{1} \leqq(n+2 \nu+1) !(\rho|\gamma|)^{-n} E_{n v}\left(a e^{\gamma z} ; \rho\right) \leqq A_{2}<\infty, \quad n \geqq 0 .
$$

Here and below constants $A$ are independent of $n$ and $z$ and may change from one inequality to another.

Proof. It suffices to prove the inequalities (7) for the case where $a=\gamma=1$. To obtain an upper bound on the degree of best rational approximation to $e^{z}$ we consider the Padé rational functions $R_{n v}(z)=P_{n v}(z) / Q_{n v}(z)$. From the known identity $[6$, p. 436]

$$
e^{z} Q_{n v}(z)-P_{n v}(z)=\frac{(-1)^{v} z^{n+v+1}}{(n+\nu) !} \int_{0}^{1} e^{t z} t^{v}(1-t)^{n} d t
$$

it follows that

$$
\left|e^{z} Q_{n v}(z)-P_{n v}(z)\right| \leqq e^{\rho} \rho^{n+v+1} \nu ! n ! /(n+\nu) !(n+\nu+1) !, \quad z \text { on } \Delta_{\rho} .
$$

Since $\lim _{n \rightarrow \infty} Q_{n v}(z)=1$ uniformly for $z$ on $\Delta_{\rho}$, we deduce that for $n$ sufficiently large

$$
\left|e^{z}-R_{n \nu}(z)\right| \leqq A_{1}\left|e^{z} Q_{n v}(z)-P_{n \nu}(z)\right| \leqq A_{2} \rho^{n} /(n+2 \nu+1) !, \quad z \text { on } \Delta_{\rho},
$$

and hence $E_{n v}\left(e^{z} ; \rho\right) \leqq A \rho^{n} /(n+2 \nu+1)$ ! for $n \geqq 0$.

To obtain the lower estimate indicated in (7) we consider the rational functions $W_{n \nu}(z)$ of respective types $(n, v)$ of best Tchebycheff approximation to $e^{z}$ on $\Delta_{\rho}$. 
Since the poles of $W_{n v}(z)$ lie in $|z|>\rho$ we may write $W_{n v}(z)=p_{n}(z) / q_{n}(z)$, where $p_{n}(z)$ is a polynomial of degree $n$ and $q_{n}(z)$ is of the form

$$
q_{n}(z)=\prod_{i=1}^{v}\left(1-\lambda_{n i} z\right)=1+\sum_{i=1}^{v} a_{n i} z^{i},
$$

where $\left|\lambda_{n i}\right|<1 / \rho$ for $i=1, \ldots, \nu$. Note that $\left|q_{n}(z)\right| \leqq 2^{v}$ for $z$ on $\Gamma_{\rho}:|z|=\rho$ and hence

$$
\begin{aligned}
E_{n v}^{2}\left(e^{z} ; \rho\right) & =\left[\max \left|e^{z}-W_{n v}(z)\right| ; z \text { on } \Gamma_{\rho}\right]^{2} \\
& \geqq(2 \pi \rho)^{-1} \int_{\Gamma_{\rho}}\left|e^{z}-W_{n v}(z)\right|^{2}|d z| \\
& \geqq(2 \pi \rho)^{-1} 2^{-2 v} \int_{\Gamma_{o}}\left|e^{z} q_{n}(z)-\not p_{n}(z)\right|^{2}|d z| .
\end{aligned}
$$

Now set

$$
\mathscr{L}_{n, k} \equiv 1 /(n+k) !+\sum_{i=1}^{\nu} a_{n i} /(n+k-i) ! .
$$

Since the $n$th section of the Taylor development for $e^{z} \phi_{n}(z)$ about $z=0$ is the polynomial of degree $n$ of least squares approximation to $e^{z} q_{n}(z)$ on $\Gamma_{\rho}$, there follows for $n \geqq v$ the inequalities

$$
\begin{aligned}
\int_{\Gamma_{\rho}}\left|e^{z} q_{n}(z)-\not p_{n}(z)\right|^{2}|d z| & \geqq \int_{\Gamma_{\rho}}\left|\sum_{k=1}^{\infty} \mathscr{L}_{n, k} z^{n+k}\right|^{2}|d z| \\
& \geqq 2 \pi \rho^{2 n+1} \sum_{k=1}^{\nu+1}\left|\mathscr{L}_{n, k}\right|^{2} \rho^{2 k} \\
& \geqq 2 \pi \rho^{2 n+1}(\nu+1)^{-1}\left(\sum_{k=1}^{\nu+1}\left|\mathscr{L}_{n, k}\right| \rho^{k}\right)^{2} ;
\end{aligned}
$$

the last inequality is a consequence of the Cauchy-Schwarz inequality. From (9) and (10) we deduce that $E_{n v}\left(e^{z} ; \rho\right) \geqq A \rho^{n} f_{n}\left(x_{n 1}, x_{n 2}, \ldots, x_{n v}\right), n \geqq v$, where $x_{n i}$ $\equiv \operatorname{Re} a_{n i}$. Hence the desired lower bound follows from (6) and the theorem is proved.

Theorem 1 implies that with each successive row of the $L_{\infty}$ Walsh array for $a e^{\gamma z}$ on $\Delta_{\rho}$ the degree of approximation by the $n$th entry of the row is improved by a factor of $1 / n^{2}$.

Estimates on the degree of best approximation by rational functions of respective types $(\nu, n)$ follow immediately from Theorem 1 and the identity

$$
\left|a e^{y z}-s_{v n}(z)\right|=\left|a^{-1} e^{-y z}-r_{n v}(z)\right| /\left|a^{-1} e^{-y z} r_{n v}(z)\right|,
$$

where $r_{n v}(z) \equiv 1 / s_{v n}(z)$. We obtain

COROLlaRY 1. For fixed $\nu$ the degree of best rational approximation $E_{v n}\left(a e^{\gamma z} ; \rho\right)$, $a \neq 0, \gamma \neq 0$, satisfies

$$
0<A_{1} \leqq(n+2 \nu+1) !(\rho|\gamma|)^{-n} E_{v n}\left(a e^{\gamma z} ; \rho\right) \leqq A_{2}<\infty, \quad n \geqq 0 .
$$


We now state our main result.

THEOREM 2. Suppose $r_{n v}(z)$ is a sequence of rational functions of respective types $(n, v), \nu$ fixed, which satisfy

$$
\left[\max \left|a e^{\gamma z}-r_{n v}(z)\right| ; z \text { on } \Delta_{\rho}\right]=o\left(\rho^{n}|\gamma|^{n} /(n+2 \nu-2) !\right) \text { as } n \rightarrow \infty \text {, }
$$

where $a \neq 0, \gamma \neq 0$. Then for $n$ sufficiently large each $r_{n v}(z)$ has at least $\nu-1$ finite poles and as $n$ becomes infinite the finite poles of the $r_{n v}(z)$ approach infinity. Furthermore, the sequence $r_{n v}(z)$ converges to ae $e^{\gamma z}$ uniformly on each bounded subset of the plane.

Proof. It suffices to prove Theorem 2 for the case where $a=\gamma=1$. If some subsequence of the $r_{n v}(z)$, say $r_{k v}(z)$, possesses $\nu-2$ or fewer finite poles, then Theorem 1 implies that

$$
\left[\max \left|e^{z}-r_{k v}(z)\right| ; z \text { on } \Delta_{\rho}\right] \geqq A \rho^{k} /(k+2(\nu-2)+1) !,
$$

which contradicts (13). Hence for $n$ large enough each $r_{n v}(z)$ has at least $\nu-1$ finite poles.

Now write $r_{n v}(z)=p_{n}(z) / q_{n}(z)$, where $p_{n}(z)$ is a polynomial of degree $n$ and $q_{n}(z)$ is the polynomial of the form $q_{n}(z)=\prod_{i=1}^{v}\left(1-\alpha_{n i} z\right)$ whose zeros are the finite poles of $r_{n v}(z)$. Note that since each $\alpha_{n i}$ is in modulus bounded by $1 / \rho$ the $q_{n}(z)$ form a normal family in the whole plane $C$. We shall show in fact that $q_{n}(z) \rightarrow 1$ uniformly on each compact subset of $C$. Let $q(z)$ be any limit function of the $q_{n}(z)$ and suppose that $q(z)$ is nonconstant in $C$. Since $q(z)$ must be a polynomial there is at least one point $\beta,|\beta| \geqq \rho$, for which $q(\beta)=0$. Now choose $\tau(>|\beta|)$ so that no zero of $q(z)$ lies on $|z|=\tau$. From the inequalities (8) and (13) it follows that

$$
\left|r_{n v}(z)-R_{n v}(z)\right| \leqq \varepsilon_{n} \rho^{n} /(n+2 \nu-2) !, \quad z \text { on } \Delta_{\rho},
$$

where $\varepsilon_{n} \rightarrow 0$ as $n \rightarrow \infty$. Hence

$$
\left|p_{n}(z) Q_{n v}(z)-P_{n v}(z) q_{n}(z)\right| \leqq A \varepsilon_{n} \rho^{n} /(n+2 \nu-2) !, \quad z \text { on } \Delta_{\rho},
$$

and since the function whose absolute value appears in (14) is a polynomial of degree $n+\nu$ the generalized Bernstein lemma [1, p. 77] implies that

$$
\left|p_{n}(z) Q_{n \nu}(z)-P_{n \nu}(z) q_{n}(z)\right| \leqq A \varepsilon_{n} \tau^{n} /(n+2 \nu-2) !, \quad|z|=\tau .
$$

Suppose now that the subsequence $q_{k}(z)$ of the $q_{n}(z)$ converges to $q(z)$ uniformly on compact subsets of $C$ and note that for $k$ large enough we have $\left|q_{k}(z) Q_{k v}(z)\right| \geqq \eta>0$ for $|z|=\tau$. Then from (15) there follows $\left|r_{k v}(z)-R_{k v}(z)\right| \leqq A \varepsilon_{k} \tau^{k} /(k+2 v-2) !,|z|=\tau$, and so from (8) (with $\rho$ replaced by $\tau$ ) and the triangle inequality we obtain

$$
\left|e^{z}-r_{k v}(z)\right| \leqq \sigma_{k} \tau^{k} /(k+2 \nu-2) !, \quad|z|=\tau,
$$

where $\sigma_{k} \rightarrow 0$ as $k \rightarrow \infty$. It is clear that for $k$ sufficiently large each $r_{k v}(z)$ has at least one pole in $\rho<|z|<\tau$, say at the point $1 / \alpha_{k}$. In [8, Lemma 1] it is shown that 
the presence of such poles $1 / \alpha_{k}$ lying interior to $|z|=\tau$ does not help to improve the degree of rational approximation on $|z|=\tau$. We briefly justify this fact.

Let $M_{k} \equiv \sigma_{k} \tau^{k} /(k+2 \nu-2) !, \pi_{k}(z) \equiv q_{k}(z) /\left(1-\alpha_{k} z\right)$ and note that from (16) we have $\left|\pi_{k}(z) e^{z}-p_{k}(z) /\left(1-\alpha_{k} z\right)\right| \leqq A M_{k},|z|=\tau$. The transformation $w=T_{k}(z) \equiv$ $\tau\left(1-\alpha_{k} z\right) / \alpha_{k}\left(\tau^{2}-z / \bar{\alpha}_{k}\right)$ maps $|z|=\tau$ onto $|w|=1$ and hence

$$
\left|T_{k}(z) \pi_{k}(z) e^{z}-S_{k}(z)\right| \leqq A M_{k}, \quad|z|=\tau,
$$

where $S_{k}(z) \equiv \tau p_{k}(z) / \alpha_{k}\left(\tau^{2}-z / \bar{\alpha}_{k}\right)$. Since the function whose absolute value appears in (17) is analytic in $|z| \leqq \tau$, the Maximum Principle implies that (17) also holds for $z=1 / \alpha_{k}$ and so $\left|S_{k}\left(1 / \alpha_{k}\right)\right| \leqq A M_{k}$. Hence

$$
\left|T_{k}(z) \pi_{k}(z) e^{z}-\left(S_{k}(z)-S_{k}\left(1 / \alpha_{k}\right)\right)\right| \leqq 2 A M_{k}, \quad|z|=\tau,
$$

from which it follows that $\left|e^{z}-s_{k-1, v-1}(z)\right| \leqq A_{1} M_{k},|z|=\tau$, where $s_{k-1, v-1}(z)$ $\equiv\left(S_{k}(z)-S_{k}\left(1 / \alpha_{k}\right)\right) / T_{k}(z) \pi_{k}(z)$ is a rational function of type $(k-1, \nu-1)$.

Clearly the above procedure may be repeated (at most $\nu-1$ additional times) to obtain a sequence of rational functions $t_{k-1, v-1}(z)$ of respective types $(k-1, v-1)$ which have no poles on $\Delta_{\tau}$ and which satisfy $\left|e^{z}-t_{k-1, v-1}(z)\right| \leqq A M_{k}, z$ on $\Delta_{\tau}$. But then $E_{k-1, v-1}\left(e^{z} ; \tau\right)=o\left(\tau^{k-1} /(k+2 \nu-2) !\right)$ as $k \rightarrow \infty$ which contradicts Theorem 1. Hence $q(z)$ must be constant in $C$ and since $q(0)=1$ we have $q(z) \equiv 1$. From the arbitrariness of $q(z)$ as a limit function of the $q_{n}(z)$ it therefore follows that $q_{n}(z) \rightarrow 1$ uniformly on each compact subset of $C$ and so the finite poles of the $r_{n v}(z)$ approach infinity.

Furthermore since $q_{n}(z) \rightarrow 1$ we can deduce inequality (16) for each $\tau(>\rho)$ and every sufficiently large integer $k$. Hence $r_{n v}(z) \rightarrow e^{z}$ uniformly on bounded subsets of $C$ which completes the proof.

From Theorems 1 and 2 we deduce

COROLlaRy 2. For fixed $\nu$ and $n$ sufficiently large, each entry $W_{n v}(z)$ of the $(\nu+1)$ th row of the $L_{\infty}$ Walsh array for the function $a e^{\gamma z}, a \neq 0, \gamma \neq 0$, on the disk $\Delta_{o}$ has precisely $\nu$ finite poles. As $n$ becomes infinite these poles approach infinity and the $W_{n v}(z)$ converge to a $e^{\gamma z}$ uniformly on each bounded subset $S$ of the plane. If $S \subset \Delta_{\tau}$, $\rho \leqq \tau<\infty$, then for $n$ sufficiently large we have

$$
\left[\max \left|a e^{\gamma z}-W_{n v}(z)\right| ; z \text { on } S\right] \leqq A \tau^{n} /(n+2 \nu+1) ! .
$$

We remark that in Theorem 2 the hypothesis concerning the degree of approximation cannot be weakened. For suppose that the points $z_{n}, n=1,2, \ldots$, are everywhere dense in $|z|>\rho$ and choose constants $B_{n}(>0)$ so small that

$$
\left|B_{n} /\left(z-z_{n}\right)\right| \leqq \rho^{n} /(n+2 \nu-2) !, \quad z \text { on } \Delta_{\rho} .
$$

Then the rational functions $r_{n v}(z) \equiv R_{n-1, v-1}(z)+B_{n} /\left(z-z_{n}\right)$, which for $n \geqq v$ are of respective types $(n, v)$, satisfy

$$
\left[\max \left|e^{z}-r_{n \nu}(z)\right| ; z \text { on } \Delta_{\rho}\right] \leqq A \rho^{n} /(n+2 \nu-2) !,
$$

and have every point of $|z|>\rho$ as a limit point of poles. We thus state for reference 
THEOREM 3. For $\nu \geqq 1$ Theorem 2 is best possible in the sense that if in (13) we replace $o\left(\rho^{n}|\gamma|^{n} /(n+2 \nu-2) !\right)$ by $O\left(\rho^{n}|\gamma|^{n} /(n+2 \nu-2)\right.$ !) then the conclusions of Theorem 2 need not hold.

From Theorem 2 and the identity (11) it is easy to deduce the following analogue of Theorem 2 for approximation by rational functions of types $(\nu, n)$ :

THEOREM 4. Suppose $s_{v n}(z)$ is a sequence of rational functions of respective types $(\nu, n), \nu$ fixed, which satisfy

$$
\left[\max \left|a e^{\gamma z}-s_{v n}(z)\right| ; z \text { on } \Delta_{\rho}\right]=o\left(\rho^{n}|\gamma|^{n} /(n+2 \nu-2) !\right) \text { as } n \rightarrow \infty \text {, }
$$

where $a \neq 0, \gamma \neq 0$. Then for $n$ sufficiently large, each $s_{v n}(z)$ has at least $v-1$ finite zeros. As $n$ becomes infinite the finite zeros and finite poles of the $s_{v n}(z)$ approach infinity and the sequence $s_{v n}(z)$ converges to a $e^{y z}$ uniformly on every bounded subset of the plane.

Consequently each column of the $L_{\infty}$ Walsh array for a $e^{\gamma z}$ on $\Delta_{\rho}$ converges to a $e^{\gamma z}$ uniformly on every bounded subset of the plane.

3. Line integral norms. The results and methods of $\$ 2$ have immediate application to approximation in the sense of least $p$ th powers on $\Gamma_{\rho}:|z|=\rho$, i.e., where the norm is defined by

$$
\|f(z)\|_{p} \equiv\left(\int_{\Gamma_{\rho}}|f(z)|^{p}|d z|\right)^{1 / p}, \quad p>0 .
$$

If $f(z)$ is continuous on $\Gamma_{\rho}$ there exists for each type $(n, v)$ a rational function $w_{n v}(z)$ of that type such that $E_{n v}^{(p)}(f ; \rho) \equiv\left\|f(z)-w_{n v}(z)\right\|_{p}$ is a minimum for all rational functions of type $(n, \nu)$. The doubly infinite table, whose entries are the best approximating rational functions $w_{n v}(z)$, is called the $L_{p}$ Walsh array for $f(z)$ on $\Gamma_{\rho}$. Concerning the degree of approximation of the rows of this array for the exponential function we have

THEOREM 5. For fixed $\nu$ the degree of best rational approximation $E_{n v}^{(p)}\left(a e^{\gamma z} ; \rho\right)$ to the function $a e^{\gamma z}, a \neq 0, \gamma \neq 0$, in the sense of least pth powers on $\Gamma_{\rho}$ satisfies

$$
0<A_{1} \leqq(n+2 \nu+1) !(\rho|\gamma|)^{-n} E_{n v}^{(p)}\left(a e^{\gamma z} ; \rho\right) \leqq A_{2}<\infty, \quad n \geqq 0 .
$$

Proof. For convenience we assume that $a=\gamma=1$. The upper bound in (18) is immediate from Theorem 1 and the inequality $E_{n v}^{(p)}\left(e^{z} ; \rho\right) \leqq(2 \pi \rho)^{1 / p} E_{n v}\left(e^{z} ; \rho\right)$.

To obtain the lower bound we assume to the contrary that for some sequence of positive integers $k$ we have

$$
(k+2 \nu+1) ! \rho^{-k} E_{k \nu}^{(p)}\left(e^{z} ; \rho\right) \rightarrow 0 \text { as } k \rightarrow \infty .
$$

Let $w_{k v}(z)$ be rational functions of respective types $(k, v)$ of least $p$ th power approximation to $e^{z}$ on $\Gamma_{\rho}$. Since

$$
\left\|e^{z}-R_{k, v+1}(z)\right\|_{p}=o\left(\rho^{k} /(k+2 \nu+1) !\right) \text { as } k \rightarrow \infty,
$$


we deduce from (19) and the modified triangle inequalities [1, p. 93] that

$$
\left\|w_{k v}(z)-R_{k, v+1}(z)\right\|_{p}=o\left(\rho^{k} /(k+2 \nu+1) !\right) \text { as } k \rightarrow \infty .
$$

Now choose polynomials $\pi_{k}(z)$ such that the zeros of $\pi_{k}(z)$ are the finite poles of $w_{k v}(z)-R_{k, v+1}(z)$ and such that the $\pi_{k}(z)$ are uniformly bounded on each compact subset of $C$. Since

$$
\left\|\pi_{k}(z)\left(w_{k v}(z)-R_{k, v+1}(z)\right)\right\|_{p}=o\left(\rho^{k} /(k+2 \nu+1) !\right) \text { as } k \rightarrow \infty,
$$

it follows by using the analogue [1, p. 92] of the Bernstein lemma that there is a subsequence $w_{k_{i}, v}(z)$ and a $\tau(>\rho)$ such that

$$
\left[\max \left|e^{z}-w_{k_{i}, v}(z)\right| ; z \text { on } \Gamma_{\tau}\right]=o\left(\tau^{k_{i}} /\left(k_{i}+2 \nu+1\right) !\right) \text { as } k_{i} \rightarrow \infty .
$$

Since the last inequality leads to a contradiction of Theorem 1 the proof is complete.

We remark that if $p \geqq 2$, then the lower estimate in (18) is immediate from the proof of Theorem 1 and from the fact that $\|f\|_{p} \geqq M\|f\|_{2}$, where $M$ is a constant dependent on $p$ and $\rho$.

The methods used to prove Theorem 2 also yield

THEOREM 6. Theorem 2 remains valid if condition (13) is replaced by the hypothesis

$$
\left\|a e^{\gamma z}-r_{n \nu}(z)\right\|_{p}=o\left(\rho^{n}|\gamma|^{n} /(n+2 \nu-2) !\right) \text { as } n \rightarrow \infty \text {. }
$$

Consequently every row of the $L_{p}$ Walsh array for a $e^{\gamma z}$ on $\Gamma_{\rho}$ converges uniformly to $a e^{y z}$ on each bounded subset of the plane.

The proof of Theorem 2 depends on the fact that with each successive row of the Walsh array for the exponential function the degree of approximation is significantly improved. It thus seems likely that the methods of this paper can be used to deduce the convergence of the rows of other Walsh arrays which have this same property. These further applications shall be reserved for a later occasion.

\section{REFERENCES}

1. J. L. Walsh, Interpolation and approximation by rational functions in the complex domain, 3rd ed., Amer. Math. Soc. Colloq. Publ., vol. 20, Amer. Math. Soc., Providence, R. I., 1960. MR 36 \#1672a.

2. - On approximation to an analytic function by rational functions of best approximation, Math Z. 38 (1934), 163-176.

3. - The convergence of sequences of rational functions of best approximation with some free poles, Proc. Sympos. Approximation of Functions, General Motors Res. Lab., 1964, Elsevier, Amsterdam, 1965, pp. 1-16. MR 32 \#4441.

4. E. B. Saff, On the row convergence of the Walsh array for meromorphic functions, Trans. Amer. Math. Soc. 146 (1969), 241-257. 
5. G. Meinardus, Approximation of functions: Theory and numerical methods, Springer Tracts in Natural Philosophy, vol. 13, Springer-Verlag, Berlin and New York, 1967. MR 36 \#571.

6. O. Perron, Die Lehre von den Kettenbrüchen, 2nd ed., Teubner, Leipzig, 1929.

7. C. Berge, Topological spaces, Macmillan, New York, 1963.

8. E. B. Saff, Approximation by rational and meromorphic functions having a bounded number of free poles, Trans. Amer. Math. Soc. 141 (1969), 79-92.

9. T. Muir, A treatise on the theory of determinants, rev. ed., Dover., New York, 1960. MR 22 \#5644.

UNIVERSITY OF SOUTH FLORIDA,

TAMPA, FLORIDA 33620 